\title{
Age Related Changes in Attentional Bias Triggered by Gaze Cues
}

\author{
Kimiko Kato*, Kazuhito Yoshizaki \\ Department of Psychology, Aichi Shukutoku University, Nagakute, Japan \\ Email: *kimi@asu.aasa.ac.jp
}

How to cite this paper: Kato, K. and Yoshizaki, K. (2019) Age Related Changes in Attentional Bias Triggered by Gaze Cues. Journal of Behavioral and Brain Science, 9, 395-405.

https://doi.org/10.4236/jbbs.2019.912030

Received: December 16, 2019

Accepted: December 20, 2019

Published: December 23, 2019

Copyright (c) 2019 by author(s) and Scientific Research Publishing Inc. This work is licensed under the Creative Commons Attribution International License (CC BY 4.0).

http://creativecommons.org/licenses/by/4.0/

\section{(c) (i) $\$$ Open Access}

\begin{abstract}
We investigated if attentional bias directed to the right increased with age. We assessed the characteristics of the following types of eye-gaze by using the Posner cueing paradigm. Younger $(n=16)$ and older $(n=20)$ adults participated in this study. First of all, a face which looked straight ahead was presented at the center of screen, followed by a gaze cue that looked left or right. Immediately after this informative cue, a target stimulus ("*”) appeared to the left or right of the face. The stimulus-onset asynchrony (SOA) between the cue and the target was selected from 300, 700, and 1100 ms. Participants were required to judge whether the target appeared to the left or the right of the gaze cue as quickly and accurately as possible. Results showed that older adults indicate a larger positive gaze-cueing effect when the eye-gaze shifted rightward, whereas this effect was not observed for a leftward shift. Moreover, a negative gaze-cueing effect (inhibition of return) was observed when the SOA was longer only for the leftward eye-gaze shift of older adults. These modulations of the cueing effect did not appear in younger adults. These findings demonstrate that the rightward attentional bias in older adults is more robust than the leftward bias.
\end{abstract}

\section{Keywords}

Aging, Visuospatial Attention, Gaze Cueing Effect, Rightward Bias

\section{Introduction}

Cerebral dominance in visual information processing differs according to the type of information, either linguistic, figural, or facial information. The laterality produced by cerebral dominance results in an attentional bias directed at the left or the right direction. Previous studies using behavioral indices and neuroimaging techniques have reported different findings regarding the laterality for dif- 
ferent types of stimuli [1]. However, changes in laterality throughout the lifespan have not been sufficiently investigated to date. Therefore, this study examined aging effects on the visuospatial attention bias.

Laterality is observed for spatial attention functions, with the right hemisphere being dominant for spatial attention [2] [3] [4]. The right and the left hemispheres control orienting towards the contralateral space. However, the right hemisphere conducts not only orientation towards the left space but also orientation towards the ipsilateral, i.e., the right space [5], which explains why most patients with unilateral neglect suffer from left-side neglect, which is caused by damage to the right hemisphere [5] [6]. In other words, even when the left hemisphere is damaged, and the orienting toward the right space deteriorates, the right hemisphere is still able to conduct orienting toward the right and left spaces. On the other hand, when the right hemisphere is damaged and orienting towards both sides deteriorates, orienting toward the left space would become impossible, although orienting towards the right space by the left hemisphere would remain possible.

Heilman et al. (1987) suggested that orienting toward the left space is performed only by the right hemisphere, whereas orienting toward the right space is performed by both the right and the left hemispheres, which results in a relatively stable orientation toward the right space [5]. However, there is little evidence of a bias towards the right in healthy adults under conditions, which direct attention to a specific stimulus to complete a task.

Recently, Takio, Koivisto, and Hämäläinen (2014) suggested a hypothetical model, in which developmental changes in executive functions throughout the lifespan produce changes in spatial bias for an imperative stimulus in visual and auditory laterality tasks [7]. Takio and colleagues conducted a visual divided spatial attention test with non-linguistic dots of light as stimuli for healthy participants aged 5 to 79 years [8] and measured visuospatial bias under three conditions: LVF condition in which dots were presented to the left visual field, RVF condition in which dots were presented to the right visual field, and BVF condition in which dots were presented to both visual fields. The results indicated that 10 - 11-year-old children and older adults over 59 years showed an RVF advantage, whereas in other age groups there were no differences between the LVF and the RVF.

Takio and colleagues' findings obtained from auditory modality (the auditory attention test [9]: the dichotic listening forced-attention test [10]) supported their hypothetical model [7]. Children with immature frontal lobe functions and older adults with reduced frontal lobe functions might not be able to control attention towards the right or the left space, which results in a bias towards the right. On the other hand, adults with mature frontal lobe functions could orient their attention appropriately based on the instructions because the frontal lobe suppressed the bias toward the right. If this explanation were correct, it could be assumed that when the load on the frontal lobe increases, even healthy adults would be unable to suppress the bias toward the right, which would result in a 
bias that is similar to children and older adults.

Strong bias to the right has been identified for both visual and auditory sensations in situations in which there is a substantial load on the frontal lobe [8] [9]. Concretely, the rightward bias tends to be exceptionally prominent when it is difficult to predict the location in which a target might be presented due to multiple possibilities, or when there is a time limit. The Simon effect for example [11], in which participants are required to respond quickly to a target presented in the right or the left visual field, is more significant when the target is presented in the right visual field [12].

The current study used the gaze cueing method [13] [14] with healthy right-handed adult and older adult participants to clarify age-related differences in the strong bias to the right area, by assessing the cueing effect and inhibition of return (IOR). A gaze cueing paradigm was developed using a modification of Posner's cueing paradigm [15]. In the original Posner's cueing method, a spatially uninformative cue such as a flash is first presented on the right or left side of the fixation point. Following a variable interval after the cue onset (SOA), a target is presented on the right or the left side of the screen. The participants are required to quickly respond to the target under one of the two conditions: when the direction of the cue is consistent with the location of the target (valid condition), or when the target is presented in the opposite location to the cue direction (invalid condition). Results indicated that when the SOA is short, responses to the target is faster in the valid than in the invalid condition, indicative of a (positive) cueing effect. On the contrary, viewers respond faster under the invalid condition compared to the valid condition under a longer SOA, which is indicative of IOR.

Previous studies have demonstrated that the modulation of the cueing effect by the SOA differs between the gaze cues and exogenous cues, such as a lateralized flash. In the typical gaze cueing paradigm, instead of a lateralized cue, a face gazing towards the left or the right is presented in the center of the screen. Compared to the experimental settings using an exogenous flash cue, for example, the positive gaze cueing effect lasts for a relatively long time. Moreover, a negative gaze cueing effect (IOR) is unlikely to be observed in the gaze cueing paradigm [14] [16].

We used a gaze cueing method in which an uninformative gaze cue was presented in the center of the screen such that the participants could not predict the target location by the cue direction. The gaze cue was presented under three SOA conditions: 300 ms-, 700 ms-, and 1100 ms-SOA. This study focused on three issues related to differences in the asymmetry of the cueing effect. It was predicted that the cueing effect of the right gaze cue would be more significant than the left gaze cue under the $300 \mathrm{~ms}-\mathrm{SOA}$ condition, and this tendency would be stronger in older compared to younger adults. The second issue was age differences in IOR asymmetry. We predicted, based on previous findings [14] [16] that IOR would not be observed in younger adults in the longer SOA condition. On the other hand, we predicted that IOR in older adults is modulated by SOA. 
Because it is possible that the decrement of frontal lobe function would lead to enhancement of the rightward bias [7], we predicted that IOR would be observed when the SOA following a left-gaze cue was over $700 \mathrm{~ms}$.

\section{Method}

\subsection{Participants}

Undergraduate students ( $n=18$, age range: 18 - 21 years, $M=19.8, S D=1.1,3$ men and 15 women) and adults ( $n=30$, age range: $65-75$ years, $M=69.1, S D=$ 2.9, 17 men and 13 women) participated in the study. Older adults were recruited from a community senior human resource center. There was no difference in the years of education between the two age groups (younger adults, $M=$ 13.6 years, $S D=1.1$; older adults, $M=13.1$ years, $S D=2.0: t(37)=0.87, p=$ 0.39). All participants gave their informed consent for taking part in this study and received a monetary reward for participation. Handedness in all the participants was assessed by the Japanese version of the FLANDERS handedness questionnaire [17]. The Mini Mental State Examination (MMSE) [18], which is a quick and easy measure for evaluating cognitive function was administered to older adults. Nine older participants were excluded from the analysis because seven were left-handed and two scored below the cutoff score $(<23 / 30)$ on the MMSE. The data of two younger adults and an older adult could not be saved due to the machine trouble. All the procedures of the study were approved by the ethics committee of Aichi Shukutoku University (No. 2014-002).

\subsection{Stimuli}

A schematic face was drawn with a black line on a white rectangle (3.6 degrees in height and 3.3 degrees in width), as shown in Figure 1. Each stimulus face measured 3.4 degrees in height and 3.2 degrees in width. The eyes measured 0.4 degrees in height and 0.9 degrees in width. The pupils subtended 0.4 degrees and

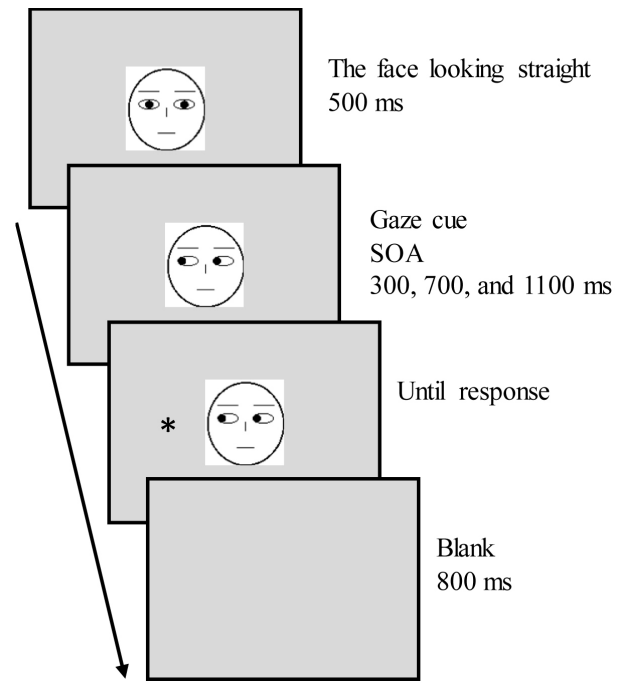

Figure 1. Illustration of the trial sequence of valid conditions. 
were either centered in the eyes (straight ahead) or both pupils just touching the left or right end of the eyes (left or right gaze). The eyebrows were 0.9 degrees, the nose was 0.4 degrees, and the mouth was 0.8 degrees in length. The target stimulus was a black asterisk that subtended 0.9 degrees. All stimuli were displayed on a gray background.

\subsection{Apparatus}

A personal computer equipped with a 24 -in. TFT monitor with a refresh rate of $144 \mathrm{~Hz}$ was used to present the stimuli. The software "SuperLab Version 5.05" (Cedrus Company, San Pedro, CA, USA) controlled the stimulus presentation and recorded the participant's responses and reaction times. The responses were recorded using a response box (Cedrus RB-530), which recorded the responses with an accuracy within $1 \mathrm{~ms}$.

\subsection{Experimental Design}

The experiment had a $2 \times 2 \times 2 \times 3$ mixed factorial design. The first factor was the age group, which consisted of the younger and the older adult groups. The second factor was gaze direction, which consisted of the left and the right gaze conditions. The third factor was the cue-target validity, which consisted of a valid condition in which a target was presented in the gaze direction and an invalid condition in which a target was presented counter to the gaze direction. The fourth factor condition was the SOA between the gaze cue and the target, consisting of $300 \mathrm{~ms}-, 700 \mathrm{~ms}-$, or $1100 \mathrm{~ms}-\mathrm{SOA}$ conditions as within-participants factors.

\subsection{Procedure}

The experiment was conducted individually in a quiet room. The viewing distance between participants' eyes and the monitor was $45 \mathrm{~cm}$, which was maintained with a chin rest. The task required participants to identify the target location. As shown in Figure 1, a trial started with a face display that portrayed a face looking straight ahead, which was presented at the center of the screen for $500 \mathrm{~ms}$. Then, the face display was replaced with a gaze cue that looked left or right. Following one of three possible gaze cue durations (the three SOA conditions), 300, 700, or $1100 \mathrm{~ms}$, the target, which was an asterisk, appeared at the left or right of the gaze cue such that it was located at 5.20 degrees to either the left or to the right of the center of the screen. Participants were required to judge whether the target appeared to the left or the right of the gaze cue as quickly and accurately as possible and respond by pressing the response button with their right index finger for a target on the right, or with their left index finger for a target on the left. The gaze cue and target remained on the screen until a response was made. All reaction times were measured from the target onset to a response. The inter-trial interval was $800 \mathrm{~ms}$. Participants were told to fixate their eyes on the face in the center of the screen, although the face was unrelated to performing the task. Following a practice block of 12 trials, participants re- 
sponded to eight experimental blocks, each with 48 trials (cue direction (left/right) $\times$ cue-target validity (valid/invalid) $\times$ SOA $(300 / 700 / 1100) \times$ four times).

\section{Results}

Following analysis used the data from 16 younger adults and 20 older adults. Demographics for participants included in the following analysis are provided in Table 1. There was no difference in the years of education between the two age groups (younger adults, $M=13.6$ years, $S D=1.1$; older adults, $M=13.4$ years, $S D=1.8: t(34)=0.40, p=0.69)$.

The mean individual reaction times for correct responses of each participant were calculated. Trials below $100 \mathrm{~ms}$ and trials above $1200 \mathrm{~ms}$ were treated as errors (younger group: 0.00\%; older group: 0.05\%). Table 2 shows the mean reaction times of younger and older adults. The mean error rates were less than $0.29 \%$ in the younger and $0.16 \%$ in the older group. Therefore, we analyzed only the reaction times for correct responses.

We conducted a $2 \times 2 \times 2 \times 3$ mixed factorial analysis of variance (ANOVA) with age group (younger and older adults) as the between-participants factor, and gaze direction (left and right), cue-target validity (valid and invalid), and SOA (100 ms, $700 \mathrm{~ms}$, and $1100 \mathrm{~ms}$ ) as within-participants factors. The results indicated main effects of age group, $F(1,34)=36.33, p<0.001, \eta_{p}^{2}=0.52$,

Table 1. Participant characteristics.

\begin{tabular}{cccc}
\hline & & Younger & Older \\
\hline Number of participants & & 16 & 20 \\
Age & Mean & 19.8 & 68.8 \\
& SD & 1.2 & 2.7 \\
Education (years) & Mean & 13.6 & 13.4 \\
& SD & 1.1 & 1.8 \\
MMSE & Mean & - & 28.9 \\
& SD & - & 1.1 \\
\hline
\end{tabular}

Table 2. Mean reaction times and SDs as a function of the experimental condition in each age group.

\begin{tabular}{cccccccccccccccc}
\hline \multicolumn{1}{c}{ Gaze direction } & \multicolumn{3}{c}{ left } & \multicolumn{4}{c}{ right } \\
\hline \multicolumn{2}{c}{ Cue-target validity } & \multicolumn{2}{c}{ valid } & \multicolumn{3}{c}{ invalid } & \multicolumn{3}{c}{ valid } & \multicolumn{4}{c}{ invalid } \\
\hline \multicolumn{2}{c}{ SOA } & 300 & 700 & 1100 & 300 & 700 & 1100 & 300 & 700 & 1100 & 300 & 700 & 1100 \\
\hline \multirow{2}{*}{ younger } & Mean & 342 & 321 & 318 & 349 & 323 & 321 & 341 & 316 & 317 & 350 & 327 & 323 \\
& SD & 36 & 33 & 31 & 37 & 31 & 34 & 34 & 26 & 32 & 37 & 32 & 35 \\
\multirow{2}{*}{ older } & Mean & 465 & 436 & 429 & 455 & 422 & 409 & 443 & 423 & 413 & 484 & 439 & 430 \\
& $S D$ & 70 & 69 & 69 & 66 & 65 & 56 & 70 & 61 & 56 & 77 & 79 & 72 \\
\hline
\end{tabular}


cue-target validity, $F(1,34)=8.87, p=0.005, \eta_{p}^{2}=0.21$, and SOA, $F(2,68)=$ $101.45, p<0.001, \eta_{p}^{2}=0.75$. The main effect of cue direction was not significant, $F(1,34)=1.67, p<0.205, \eta_{p}^{2}=0.05$. Moreover, there were two-way interactions of age $\times$ SOA, $F(2,68)=4.94, p=0.010, \eta_{p}^{2}=0.13$, gaze direction $\times$ cue-target validity, $F(1,34)=6.72, p=0.014, \eta_{p}^{2}=0.17$, and cue-target validity $\mathrm{x}$ SOA, $F(2,68)=6.07, p=0.004, \eta_{p}^{2}=0.15$, whereas the other two-way interactions did not reach significance, $F s<1.70$, $p s>0.20$.

A significant three-way interaction, (age group $\times$ gaze direction $\times$ cue-target validity, $\left.F(1,34)=4.14, p=0.050, \eta_{p}^{2}=0.11\right)$ was observed, which suggested that the reaction time was faster in older adults for the valid compared to the invalid condition of the right gaze, indicative of a positive cueing effect, $F(1,68)$ $=14.19, p<0.001, \eta_{p}^{2}<0.17$, whereas the left gaze of older adults showed IOR, or a negative cueing effect, $F(1,68)=4.89, p=0.030, \eta_{p}^{2}=0.07$. However, younger adults did not show a significant difference between valid and invalid conditions in either gaze direction, the left gaze, $F(1,68)=0.33, p=0.567, \eta_{p}^{2}$ $=0.005$; the right gaze, $\left.F(1,68)=1.68, p=0.199, \eta_{p}^{2}=0.024\right)$. Also, a significant three-way interaction (age group $\times$ cue-target validity $\times \mathrm{SOA}), F(2,68)=$ 3.16, $p=0.049, \eta_{p}^{2}=0.09$, was observed, which indicated that in older adults, the positive cueing effect was observed only for the SOA 300-ms condition $\left(\right.$ SOA300-ms, $F(1,102)=18.11, p<0.001, \eta_{p}^{2}=0.15$; SOA700-ms, $F(1,102)=$ $0.08, p=0.780, \eta_{p}^{2}<0.001$; SOA1100-ms, $F(1,102)=0.12, p=0.736, \eta_{p}^{2}=$ $0.001)$. In young adults, the positive cueing effect was observed in the SOA $300-\mathrm{ms}, F(1,102)=4.51, p=0.036, \eta_{p}^{2}=0.042$, and the SOA $700-\mathrm{ms}$ conditions, $F(1,102)=2.93, p=0.090, \eta_{p}^{2}=0.028$, but not in the SOA $1100-\mathrm{ms}$ condition, $F(1,102)=1.49, p=0.022, \eta_{p}^{2}=0.014$.

There was a significant four-way interaction, $F(2,68)=3.21, p=0.047, \eta_{p}^{2}=$ 0.09. To clarify this interaction, an ANOVA was conducted on the cueing effect. We calculated the cueing effect by subtracting valid from invalid reaction times. We conducted a $2 \times 2 \times 3$ mixed factorial ANOVA with age group (younger and older adults) as the between-participants factor, and gaze direction (left and right) and $\operatorname{SOA}(300,700$, and 1100) as the within-participants factors. This analysis showed a significant main effect of the gaze direction, $F(1,34)=6.73, p$ $=0.014, \eta_{p}^{2}=0.17$, indicating that the cueing effect was larger for the right (17 $\mathrm{ms})$ than for the left $(-5 \mathrm{~ms})$ gaze conditions. The main effect of SOA was also significant, $F(2,68)=6.07, p=0.004, \eta_{p}^{2}=0.15$. A post hoc Tukey's HSD test demonstrated that the cueing effect was larger in the 300-ms SOA condition (12 $\mathrm{ms}$ ) than in the $1100-\mathrm{ms}$ SOA condition $(2 \mathrm{~ms})$ or in the $700-\mathrm{ms}$ SOA condition (4 ms) $(p<0.05)$, whereas there was no significant difference in the cueing effect between the 700-ms and the 1100-ms SOA conditions.

There were significant two-way interactions (age group $\times$ gaze direction, $F(1$, $34)=4.14, p=0.050, \eta_{p}^{2}=0.11$; age group $\times$ SOA interaction, $F(2,68)=3.16$, $\left.p=0.049, \eta_{p}^{2}=0.085\right)$. More importantly, a significant three-way interaction between age group, gaze direction and SOA was also found, $F(2,68)=3.21, p=$ $0.047, \eta_{p}^{2}=0.086$. As can be seen in Figure 2, the simple interaction was sig- 
nificant only in the SOA 300 condition, $F(1,102)=7.145, p=0.009, \eta_{p}^{2}=$ 0.065 , reflecting that while the cueing effect was invariant across the gaze directions in younger adults, $F(1,102)=0.044, p=0.835, \eta_{p}^{2}<0.001$, it was larger for the right $(41 \mathrm{~ms})$ than for the left $(-10 \mathrm{~ms})$ gaze condition in older adults, $F(1,102)=15.915, p<0.001, \eta_{p}^{2}=0.135$. The simple interactions of other SOA conditions did not reach significance, $F s<3.485, p s>0.06$.
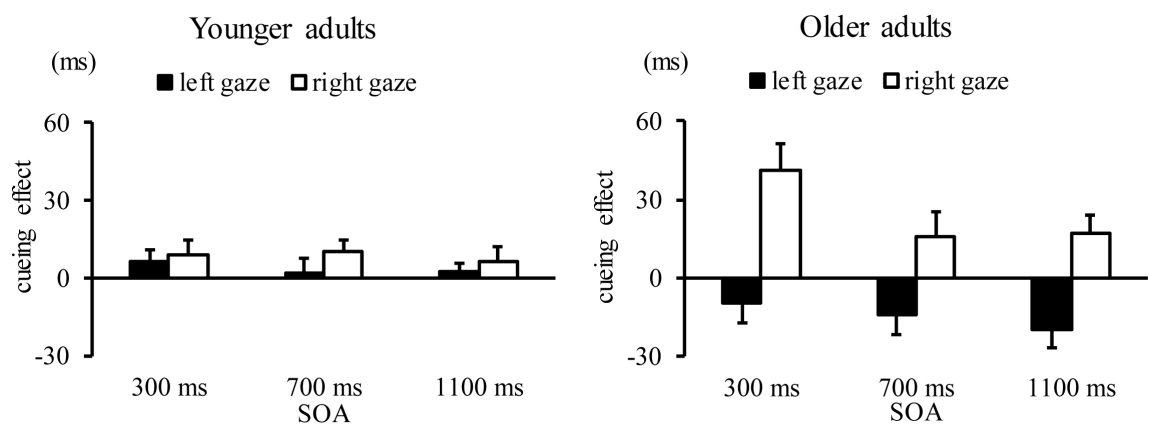

Figure 2. Mean cueing effects in each experimental condition. Bars indicate standard errors.

We focused on the modulation of the cueing effect and IOR as a function of SOAs in different age groups, by using the reaction times for making correct responses to test the predictions of this study. As can be seen in Figure 2, the cueing effect was significant in all SOA conditions (the 300-SOA condition $=41$ ms, $t(19)=3.957, p<0.001, r=0.672$; the 700-SOA condition $=16 \mathrm{~ms}, t(19)=$ $1.679, p=0.109, r=0.360$; the 1100-SOA condition $=17 \mathrm{~ms}, t(19)=2.408, p=$ $0.026, r=0.484)$ for the right gaze condition of the older group. On the contrary, in the left gaze condition of the older group, a negative cueing effect, i.e., IOR increases as the SOA is longer (300-SOA condition $=-10 \mathrm{~ms}, t(19)=1.256, p=$ $0.224, r=0.277$; 700-SOA condition $=-14 \mathrm{~ms}, t(19)=1.816, p=0.085, r=$ $0.384 ; 1100-$ SOA condition $=-20 \mathrm{~ms}, t(19)=2.778, p=0.012, r=0.537)$.

However, the modulation pattern of the cueing effect as a function of SOAs was different in the younger group from the older group. In the younger group, the cueing effect appeared only in the 700-SOA condition for the right gaze direction (300-SOA condition of right gaze, $9 \mathrm{~ms}, t(15)=1.557, p=0.140, r=$ 0.336 ; 700-SOA condition $=10 \mathrm{~ms}, t(15)=2.380, p=0.031, r=0.479 ; 1100-\mathrm{SOA}$ condition $=6 \mathrm{~ms}, t(15)=1.042, p=0.314, r=0.232)$, and the cueing effect for the left gaze was not significant in any of the SOA conditions (300-SOA condition $=6 \mathrm{~ms}, t(15)=1.432, p=0.173, r=0.312 ; 700-\mathrm{SOA}$ condition $=2 \mathrm{~ms}, t(15)$ $=0.403, p=0.693, r=0.092 ; 1100-\mathrm{SOA}$ condition $=3 \mathrm{~ms}, t(15)=0.882, p=$ $0.392, r=0.198)$. These findings indicate that the IOR did not appear in any of the three SOA conditions in the younger group, irrespective of gaze directions, which was different from older adults.

\section{Discussion}

This study used the gaze cueing effect observed in a Posner type cueing task to 
investigate whether the rightward attentional bias increased with age. Positive and negative cueing effects were used, which demonstrated for the first time that attention in older adults shifted toward the right field because the right gaze cue in the short SOA condition of $300 \mathrm{~ms}$ showed a positive gaze-cueing effect in older adults. This attentional shift toward the right visual space could be due to older adults' increased responsiveness to right gaze cues. Also, the same pattern of results observed in the short SOA $(300 \mathrm{~ms})$ condition was observed in the longer SOA conditions. These findings suggest that attention in older adults might not disengage once it is triggered by right-gaze moves toward the right space. The negative gaze-cueing effect (IOR) under the left-gaze cue indicated an attentional bias toward the right, whereas the left-gaze did not trigger a leftward attentional shift. Moreover, the attentional shift towards the right was pronounced in longer SOA conditions. These results suggested that older adults are deficient in engaging attention to the left field, and are likely to shift their attention to the right field. The results of younger adults indicated that a positive gaze-cueing effect triggered by a gaze bias toward the right side similar to older adults was only in the $700 \mathrm{~ms}$-SOA condition. Our results generally supported previous findings in which an endogenous cue resulted in the maximal cue effect with SOA of over $300 \mathrm{~ms}$ [19] [20].

The finding that attentional bias toward the right was observed only in older adults could be interpreted according to the contention by Takio et al. (2014) [7]. According to Takio et al., younger adults can control their attention toward the right or left space equally well, because they have good frontal lobe functions. However, in older people, the age-related decrease of frontal lobe functions might also deteriorate attentional control, which might cause the right bias in the attentional orientation of older adults.

Another interpretation of the rightward shift in visuospatial attention of older adults could be the age-related changes in brain activity that reduce hemispheric asymmetry for memory tasks, as suggested by neuroimaging research [21]. Age-related changes in prefrontal activity might reflect functional compensation in older adults, which is known as hemispheric asymmetry reduction (HAROLD) [21] [22] [23]. Using the HAROLD model to explain our results of visuospatial attention suggest that a compensatory function similar to hemispheric asymmetry reduction might be observed in visuospatial attention. Reduction of right hemisphere dominance in attentional functions could deteriorate the orientation of attention to the left and right visual space. As a result, the activation of the left hemisphere might become enhanced to compensate for the reduction in visuospatial function of the right hemisphere, which might cause a robust rightward bias. It is suggested that future research should investigate the applicability of HAROLD model to high-level cognitive functions other than memory.

It remains an open question whether the rightward bias observed in older adults would also result from endogenous cues with (gaze) or without social signification (e.g., arrow). Future study is also required to investigate whether or 
not exogenous cues trigger a rightward bias in older adults. In addition, the bias to the right in wider age groups should be investigated in further research.

This study demonstrated an age-related enhancement of the right bias in visuospatial attention that might be caused by an innate bias, or by the development of the left hemisphere function, which is associated with the reduction of visuospatial attention in the right hemisphere.

\section{Acknowledgements}

This research was supported in part by a program grant from Aichi Shukutoku University (16TT07) and by the Japan Society for the Promotion of Science KAKENHI; Grant Number JP16K04436 to Kimiko Kato and JP15K04198 to Kazuhito Yoshizaki. The authors report no conflicts of interest with the findings of this study. The study was approved by the institutional ethics committee of Aichi Shukutoku University according to the 2008 declaration of Helsinki.

\section{Conflicts of Interest}

The authors declare no conflicts of interest regarding the publication of this paper.

\section{References}

[1] Bartolomeo, P. and Seidel Malkinson, T. (2019) Hemispheric Lateralization of Attention Processes in the Human Brain. Current Opinion in Psychology, 29, 90-96. https://doi.org/10.1016/j.copsyc.2018.12.023

[2] Corbetta, M., Miezin, F.M., Shulman, G.L. and Petersen, S.E. (1993) A PET Study of Visuospatial Attention. Journal of Neuroscience, 13, 1202-1226. https://doi.org/10.1523/JNEUROSCI.13-03-01202.1993

[3] Corbetta, M. and Shulman, G.L. (2011) Spatial Neglect and Attention Networks. Annual Review of Neuroscience, 34, 569-599. https://doi.org/10.1146/annurev-neuro-061010-113731

[4] Thiebaut de Schotten, M.T., Dell'Acqua, F., Forkel, S.J., Simmons, A., Vergani, F., Murphy, D.G.M. and Catani, M. (2011) A Lateralized Brain Network for Visuospatial Attention. Nature Neuroscience, 14, 1245-1246. https://doi.org/10.1038/nn.2905

[5] Heilman, K.M., Bowers, D., Valenstein, E. and Watson, R.T. (1987) Hemispaceand Hemispatial Neglect. In: Jeannerod, M., Ed., Neurophysiological and Neuropsychological Aspects of Spatial Neglect, Elsevier Science, Amsterdam, 115-150. https://doi.org/10.1016/S0166-4115(08)61711-2

[6] Corbetta, M., Patel, G. and Shulman, G.L. (2008) The Reorienting System of the Human Brain: From Environment to Theory of Mind. Neuron, 58, 306-324. https://doi.org/10.1016/j.neuron.2008.04.017

[7] Takio, F., Koivisto, M. and Hämäläinen, H. (2014) The Influence of Executive Functions on Spatial Biases Varies during the Lifespan. Developmental Cognitive Neuroscience, 10, 170-180. https://doi.org/10.1016/j.dcn.2014.09.004

[8] Takio, F., Koivisto, M., Tuominen, T., Laukka, S.J. and Hämäläinen, H. (2013) Visual Rightward Spatial Bias Varies as a Function of Age. Laterality, 18, 44-67. https://doi.org/10.1080/1357650X.2011.628675

[9] Takio, F., Koivisto, M., Laukka, S.J. and Hämäläinen, H. (2011) Auditory Rightward 
Spatial Bias Varies as a Function of Age. Developmental Neuropsychology, 36, 367-387. https://doi.org/10.1080/87565641.2010.549984

[10] Takio, F., Koivisto, M., Jokiranta, L., Rashid, F., Kallio, J., Tuominen, T., Hämäläinen, H., et al. (2009) The Effect of Age on Attentional Modulation in Dichotic Listening. Developmental Neuropsychology, 34, 225-239. https://doi.org/10.1080/87565640902805669

[11] Simon, J.R. (1990) The Effects of an Irrelevant Directional Cue on Human Information Processing. In: Proctor, R.W. and Reeve, T.G., Eds., Stimulus-Response Compatibility: An Integrated Perspective, North-Holland, Amsterdam, 31-86. https://doi.org/10.1016/S0166-4115(08)61218-2

[12] Tagliabue, M., Vidotto, G., Umiltà, C., Altoè, G., Treccani, B. and Spera, P. (2007) The Measurement of Left-Right Asymmetries in the Simon Effect: A Fine-Grained Analysis. Behavior Research Methods, 39, 50-61.

https://doi.org/10.3758/BF03192843

[13] Friesen, C.K. and Kingstone, A. (1998) The Eyes Have It! Reflexive Orienting Is Triggered by Nonpredictive Gaze. Psychonomic Bulletin \& Review, 5, 490-495. https://doi.org/10.3758/BF03208827

[14] Frischen, A., Bayliss, A.P. and Tipper, S.P. (2007) Gaze Cueing of Attention: Visual Attention, Social Cognition, and Individual Differences. Psychological Bulletin, 133, 694-724. https://doi.org/10.1037/0033-2909.133.4.694

[15] Posner, M.I. (1980) Orienting of Attention. Quarterly Journal of Experimental Psychology, 32, 3-25. https://doi.org/10.1080/00335558008248231

[16] Driver, J., Davis, G., Ricciardelli, P., Kidd, P., Maxwell, E. and Baron-Cohen, S. (1999) Gaze Perception Triggers Reflexive Visuospatial Orienting. Visual Cognition, 6, 509-540. https://doi.org/10.1080/135062899394920

[17] Okubo, M., Suzuki, H. and Nicholls, M.E.R. (2014) A Japanese Version of the Flanders Handedness Questionnaire. Japanese Journal of Psychology, 85, 474-481. https://doi.org/10.4992/jipsy.85.13235

[18] Folstein, M.F., Folstein, S.E. and McHugh, P.R. (1975) "Minimental State": A Practical Method for Grading the Cognitive State of Patients for the Clinician. Journal of Psychiatric Research, 12, 189-198. https://doi.org/10.1016/0022-3956(75)90026-6

[19] Cheal, M. and Lyona, D. (1989) Attention Effects on Form Discrimination at Different Eccentricities. Quarterly Journal of Experimental Psychology Section A: Human Experimental Psychology, 41, 719-746. https://doi.org/10.1080/14640748908402391

[20] Müller, H.J. and Rabbitt, P.M. (1989) Reflexive and Voluntary Orienting of Visual Attention: Time Course of Activation and Resistance to Interruption. Journal of Experimental Psychology: Human Perception and Performance, 15, 315-330. https://doi.org/10.1037/0096-1523.15.2.315

[21] Cabeza, R. (2002) Hemispheric Asymmetry Reduction in Older Adults: The Harold Model. Psychology and Aging, 17, 85-100. https://doi.org/10.1037/0882-7974.17.1.85

[22] Cabeza, R., Grady, C.L., Nyberg, L., McIntosh, A.R, Tulving, E., Kapur, S., Craik, F.I., et al. (1997) Age-Related Differences in Neural Activity during Memory Encoding and Retrieval: APositron Emission Tomography Study. Journal of Neuroscience, 17, 391-400. https://doi.org/10.1523/JNEUROSCI.17-01-00391.1997

[23] Zysset, S., Schroeter, M.L., Neumann, J. and von Cramon, D.Y. (2007) Stroop Interference, Hemodynamic Response and Aging: An Event-Related fMRI Study. Neurobiology of Aging, 28, 937-946. https://doi.org/10.1016/j.neurobiolaging.2006.05.008 